\title{
Self-Care Support for Patients with Gastrointestinal Cancer: iCancerHealth
}

\author{
Donna L. Berry ${ }^{1}$ Traci M. Blonquist ${ }^{1}$ Manan M. Nayak ${ }^{1} \quad$ Nina Grenon ${ }^{1}$ Thaer G. Momani ${ }^{2}$ \\ Nadine J. McCleary ${ }^{1}$
}
${ }^{1}$ Department of Medical Oncology, Dana-Farber Cancer Institute, Boston, Massachusetts, United States
2 Department of Medical Oncology, University of Massachusetts Boston, Boston, Massachusetts, United States

Address for correspondence Donna L. Berry, PhD, RN, AOCN, FAAN, Dana-Farber Cancer Institute, 450 Brookline Avenue, LW 518, Boston, MA 02215 (e-mail: donna_berry@dfci.harvard.edu).

Appl Clin Inform 2018;9:833-840.

\begin{abstract}
Keywords

- cancer

- symptoms

- mHealth

- telehealth

- communication

Background Patient-centered symptom assessment and management tools allow patients to perform self-assessments and engage in self-symptom management. Efficacious tools exist for reducing symptom distress; however, little is known about feature-specific use.

Objectives This article evaluates the feasibility of the iCancerHealth app as an adjunct to usual patient education regarding cancer symptoms and medication management. Methods We conducted a single-arm, pilot study grounded in the health outcomes model. Our evaluation included (1) enrollment rates, (2) 2-month utilization rates, (3) patient acceptability, and (4) clinician satisfaction with the provider-side application. English-speaking, adult patients receiving care in the gastrointestinal oncology service of a comprehensive cancer center were invited to participate. Research coordinators enrolled consenting participants who had a personal, Internet-connected device; participants registered and used the platform to complete the baseline symptom assessment in clinic. Participants were reminded weekly to use the app and to perform a symptom report 4 to 6 weeks later.

Results A total of 64 patients were approached, of which 57 (89\%; 95\% exact confidence interval [Cl], 79-96\%) enrolled. About half were $\geq 60$ years old and $40 \%$ were women. Fifty-three patients (93\%; 95\% exact $\mathrm{Cl}, 85-99 \%$ ) accessed at least one app feature, at least once, from home. The most frequently used (86\%) feature was Health Tracker in which participants monitored and reported symptoms; followed by My Inbox (63\%) and My Medications features (60\%). The mean acceptability score was 24.8 (standard deviation $=4.2$ ), indicating good acceptability. Clinicians reported that the app was most acceptable with regard to facilitating in-person interactions that occurred after app use.

Conclusion In a sample of adults with various stages of gastrointestinal malignancies, the iCancerHealth app was utilized at a high rate. Features that focused on symptoms and medication side effects plus communication with clinicians were used most frequently. This extends our understanding of preferences and specific feature use with patient-centered technologies.
\end{abstract}

received

June 11, 2018

accepted after revision

September 23, 2018 (c) 2018 Georg Thieme Verlag KG Stuttgart · New York
DOI https://doi.org/

$10.1055 / \mathrm{s}-0038-1675810$. ISSN 1869-0327. 


\section{Background and Significance}

Patients with cancer arrive in the therapeutic setting with varying levels of symptomatology and other types of responses to the cancer diagnosis and experience. Once treatment begins, another profile of symptoms commences as toxicities and treatment-related complications develop. These symptoms and the self-care required at home, and the related psychological, social, family, and work impact of cancer and its treatment, can be burdensome or overwhelming to many patients. ${ }^{1,2}$ An exhaustive systemic review ${ }^{3}$ documented cancer symptoms during active treatment as quite prevalent and severe.

Assessing and managing cancer symptoms and side effects (SX) is a major goal for both patients and oncology clinicians. Standardized assessments of SX have been developed and used widely in research studies to measure outcomes of treatments and interventions. Assessments also have been used clinically, and been found to: (1) increase the depth and breadth of discussions of SX, ${ }^{1}$ and patientreported emotional well-being ${ }^{4}$; (2) increase treatment of psychosocial issues and symptoms in patients in oncology ${ }^{4}$; and (3) reduce cancer symptom distress when combined with self-care patient education and monitoring. ${ }^{2}$

As oral chemotherapies rapidly expand in oncology practice, ${ }^{5}$ an increasingly significant concern involves patient medication adherence when these oral agents are self-administered at home. Suboptimal or improper self-administration, ${ }^{6}$ reduces treatment efficacy and increases toxicity ${ }^{7}$ and leads to treatment delays, changes in treatment, and premature death. ${ }^{8}$ Patients require systematic support for proper dosing and administration. ${ }^{9}$ With new information technologies, assessments can increasingly be conducted with computerized assessment, eliminating abstraction and data entry of paper forms, increasing accuracy of reports, making summaries available to clinicians in real time, and facilitating electronic communication. An SX assessment and management tool that is patient-centered may allow patients to perform self-assessments, learn about SX management and self-administered medications, and be coached in communicating with clinicians. An intervention that combines evidence-based instructions, reminders, and monitoring tools summaries delivered to clinicians with secured messaging may be a powerful tool in the management of patients' SX concerns.

\section{Cancer Symptoms}

Cancer symptoms not only are indications of the physiologic changes associated with disease and treatment toxicity, but also reflect linkages to patients' perceived reality, including social, psychological, and cultural factors. ${ }^{10}$ With the incidence of individual and groups of cancer symptoms ${ }^{3}$ and psychosocial distress ${ }^{11}$ at such a high levels across various diagnoses and stages and the apparent impact of such experiences on the dimensions of quality of life, the consequences of inadequate symptom management are complex and can be overwhelming to patients and their caregivers. For example, severe mucositis or radiation-associated skin changes can put patients at risk for additional complications. ${ }^{12}$ Early screening for psychosocial distress may enable clinicians to identify patients at higher risk and intervene to prevent development of crisis events, notably in those diagnoses with the greatest symptom burden ${ }^{13}$.

Making cancer symptom issues visible and discussed in the clinic can promote partnership between clinicians and patients, validating the patients' experiences and enhancing communication and satisfaction. Rapid, predictive screening may help reduce unnecessary health care utilization costs and prolonged medical treatments, as well as enhance quality of life. The challenge of efficient, systematic, and meaningful assessment is important and in current clinical cancer settings, the American College of Surgeons Committee on Cancer has set a standard for all accredited health care institutions. ${ }^{14}$ As reviewed by Mooney et al, ${ }^{15}$ randomized trials provide evidence that self-monitoring of cancer symptoms and associated communication coaching result in lower symptom distress and better patient-clinician communication. When clinicians are unaware of SX, particularly treatment-related toxicities, there is danger of higher morbidity and even mortality related to unintentional overdosing. ${ }^{12,16}$ Interventions to improve patient-clinician communication have been tested with positive results. ${ }^{1,17-20}$

\section{Medication Self-administration}

Patients require systematic support for proper dosing and administration. ${ }^{8}$ Systematic reviews of interventional studies for medication adherence support a standardized multimethod approach to medication management that involves tailored cognitive educational approaches ${ }^{21}$ with psychosocial support strategies. ${ }^{22}$ Additional use of written information and feedback and monitoring by nurses, ${ }^{23,24}$ including management of SX, can provide educational reinforcement.

\section{Usability and Acceptability}

Most people have encountered usability difficulties in their everyday lives such as trying to set the clock on the video player, ordering something online, and at self-check-outs at libraries and grocery stores. Each of these tasks requires an interaction between a human and a computer interface. If the interface is well designed, the application may be quickly embraced by the end user; however, poor design cannot only turn away potential users, but lead to measurement error and nondiffusion among target user groups. There is a wellestablished body of research in usability and structured software design methods, ${ }^{25}$ and now it has been systematically applied to the development of patient-centered software in health care venues. ${ }^{26}$ Remote utilization rates of Web-based applications have varied from 34 (consecutive clinic approaches) to $77 \%$ (self-selected volunteers) for unprompted use after enrollment in cancer symptom studies. ${ }^{2,27}$ Acceptability has typically been adequate; however, the use of a variety of different measures by various investigative teams precludes comparison.

The purpose of this pilot, feasibility study was to evaluate a unique clinical deployment of a computerized patient assessment of cancer SX, targeting a customized, patientcentered intervention to coach the patient regarding communicating priority SX plus medication self-administration prompts and patient-clinician communication messaging. 


\section{Objectives}

Our three objectives were to: (1) evaluate the feasibility of iCancerHealth as an adjunct to usual patient education regarding cancer symptoms and medication management, with regard to (i) enrollment rates and (ii) utilization over a 2-month period; (2) describe patient acceptability of iCancerHealth in general and the specific features; and (3) explore clinician satisfaction with the iCancerHealth provider-side application.

\section{Methods}

Eligible patient participants were 18 years or older and had a diagnosis of any stage malignant gastrointestinal (GI) disease, were receiving or planning treatment in the GI clinic, spoke and read English, and had Internet/cellular access via either a personal computer Web browser, iOS device (smartphone or tablet), or Android (phone only). Patients were excluded from enrollment given a documented diagnosis of a psychiatric depressive or cognitive impairment. Eligible clinician participants were nurses, physicians, or physician assistants who performed consults/exams in the GI oncology clinic.

This was a single-arm, pilot study to quantify the feasibility and acceptability of the iCancerHealth intervention. The app was provided free of charge to the investigators from the vendor, Medocity, Inc. Native apps for iOS and Android were available, plus a Web-based version for personal computer browser access.

Recruitment letters describing the elements of consent was sent to clinicians. Interested clinicians were: (1) oriented to the application (app), (2) were asked to inform eligible patients about the study during consultation, and (3) were asked to complete satisfaction survey at the end of the study. Clinicians were not compensated for participating in the study. Clinicians provided interested participants contact information to the research coordinator, who approached the potential participant and obtained written consent. All participants were provided with the opportunity to ask questions and if not comfortable to opt out. Once written consent was obtained, the research coordinator (1) helped the patient participant download the app and register an account, (2) oriented the participant to all sections of the app, and (3) collected 1st baseline (T1) SX assessment within the app. Patient participants were asked to use the app regularly for up to 6 weeks and the research staff followed up weekly in person or through a telephone call. At the 4 to 6 weeks' clinic visit, the last assessment (T2) was completed and the patient participant was given a $\$ 50$ gift card for their time and effort. Clinical characteristics including diagnosis, stage, and treatment regimen were abstracted from the patients' medical record.

For patient participants, iCancerHealth app features included real-time charting of reported symptom experiences followed by clinician-approved self-care recommendations based on the severity of symptoms. For severe symptoms, participants were instructed to contact the clinician's office right away. Education to nutrition and fluid management were included. A feature focused on medication management and adherence was provided with autoreminder notifications. The calendar feature allowed entry of appointments and other events. A community forum for peer support also was available for participants. Participants were able to send secured messages through the app to the participating clinicians. The provider side of the app provided alerts for patient participant's SX at a predetermined threshold. Clinicians were expected to respond to the alerts and read the secured messages on a daily basis. iCancerHealth was designed to be compliant with the Health Insurance Portability and Accountability Act. All messaging and data were sent through encrypted channels. A new version of the iCancerHealth app was released toward the end of the study and we marked that time point for future analyses. Modules and tabs were reorganized or renamed in the app, the three participants who were actively using versions 1 were oriented to using the new version.

Based on our previous experience with remote access to a symptom reporting and self-care instruction intervention in which $34 \%$ voluntarily (unprompted) accessed the Web-based application, ${ }^{28}$ we considered use of iCancerHealth feasible if at least a $34 \%$ remote access rate was reached. The remote access rate was defined as accessing and using iCancerHealth from a nonclinic location at least once. Additionally, we considered enrollment to iCancerHealth feasible if at least $80 \%$ of the approached GI oncology patients participated.

A total of 70 patient participants were planned for this feasibility study and a $15 \%$ attrition rate was expected. With 60 evaluable patient participants and complete T1-T2 data, the $95 \%$ confidence interval $(\mathrm{CI})$ was planned to be no wider than $26 \%$.

\section{Outcomes}

In a clinical cancer setting, all patient outcomes have been mediated by some aspect(s) of the system and/or some patient aspect(s). Modes of patient engagement with selfcare, including symptom monitoring and clinician response to notices from patients about high symptom burden, are applicable to the Health Outcomes Model, explicated by Mitchell et $\mathrm{al}^{29}$ we selected outcomes that would begin to explain variables that ultimately can inform larger trials to test patient quality of life and intervention outcomes.

Enrollment rates: Eligible patients approached to participate in the study were tracked through a secure database that documented the number of consenting patients, reasons for refusal to participate, and attrition.

Remote access and utilization rates: Use was defined as accessing app and clicking in any feature at least once. Utilization was recorded electronically in the iCancerHealth app that tracked the number of times a patient clicked on a given feature (calendar, community, dashboard, health tracker, medical diary, inbox, medications, profile, nutrition, scrapbook, or settings). The rate of utilization was defined as the proportion of patients accessing and clicking on a feature in iCancerHealth at least once after the on-study demonstration. A remote access and utilization rate of at least 34\% would indicate feasibility of iCancerHealth patient usage.

Acceptability (T2): All patient participants were presented six items on the Acceptability E-scale ${ }^{30}$ plus feature-specific items at the conclusion of the T2 iCancerHealth assessment. 
The items focused on how easy, how understandable, how enjoyable, how helpful, acceptable amount of time, and overall satisfaction with the app. Possible responses ranged from 1 to 5, with 1 indicating a low level of agreement regarding acceptability of the program and 5 indicating a high level. An average summed score of $\geq 24$ is considered adequate acceptability. The Acceptability E-scale has been found to have high consistency and reliability and has been used in over 2,000 patients with cancer. ${ }^{1,31,32}$

Clinician satisfaction (end of study): A questionnaire adapted from the Acceptability E-scale was used to assess clinician satisfaction relevant to how easy, how understandable, acceptable amount of time, overall satisfaction, followed by whether the app identified appropriate issues, facilitated electronic interactions, facilitated in-person interactions, and symptom notifications promoted communication. Clinicians were asked to provide additional feedback with one open-ended item.

\section{Analysis}

Descriptive statistics were used to summarize demographic and survey data. The enrollment rate and utilization rate were estimated and reported with an exact 95\% CI. Additionally, the utilization of each feature was described. The total score for patients answering the Acceptability E-scale was summarized (median/range, mean/standard deviation [SD]). The number of clinicians indicating a score of at least 4 on a given Acceptability E-scale item was tabled. Because a new version of the iCancerHealth app was released during the study, all analyses were also summarized by version.

\section{Results}

\section{Patient Participant Results}

A total of 64 patients were approached to participate in the study of which 57 (89\%; 95\% exact CI, 79-95\%) enrolled. Reasons given for declined participation included: too much of a time commitment $(n=1)$, does not use technology to manage care $(n=2)$, participant uses other technology for care $(n=2)$, and unknown for 2 participants. Of the 57 patients enrolled, 43 (75\%) used version one exclusively, 11 (19\%) used version two exclusively, and 3 (5\%) used both versions. Enrollment was halted early shortly after the release of a second version of the app because of difficulties with the provider interface.

Overall participant demographics are outlined in - Table 1. All participants received chemotherapy (injectable, infusion, and/or oral) with 44 (77\%) receiving infusion chemotherapy alone or in combination. A majority (84\%) of participants indicated a preference for email as the way to receive health-related reminders. Participants were allowed to select multiple preferences and other selected methods included: 29 (51\%) text message, 15 (25\%) telephone, and 2 (4\%) other (secure email and "I like the personal touch").

Participant feature utilization is displayed in -Table 2. Overall, 53 participants (93\%; 95\% exact CI, 83-98\%) used a minimum of one feature at least once. Of those with version 1 ( $n=43$ ), 41 (95\%; 95\% exact CI, 84-99\%) used the app. Of those
Table 1 Participant demographics $(N=57)$

\begin{tabular}{|c|c|c|}
\hline & $N$ & $\%$ \\
\hline \multicolumn{3}{|l|}{ Age group (y) } \\
\hline$\leq 49$ & 9 & 15.79 \\
\hline $50-59$ & 19 & 33.33 \\
\hline$\geq 60$ & 29 & 50.88 \\
\hline Non-Hispanic ${ }^{a}$ & 56 & 98.25 \\
\hline \multicolumn{3}{|l|}{ Gender } \\
\hline Male & 33 & 57.89 \\
\hline Female & 23 & 40.35 \\
\hline \multicolumn{3}{|l|}{ Race/Ethnicity } \\
\hline Asian & 3 & 5.26 \\
\hline Black & 3 & 5.26 \\
\hline White & 50 & 87.72 \\
\hline \multicolumn{3}{|l|}{ Working } \\
\hline No & 27 & 47.37 \\
\hline Yes & 30 & 52.63 \\
\hline \multicolumn{3}{|l|}{ Married/Partnered } \\
\hline No & 16 & 28.07 \\
\hline Yes & 40 & 70.18 \\
\hline \multicolumn{3}{|l|}{ Computer use } \\
\hline Sometimes & 6 & 10.53 \\
\hline Often & 12 & 21.05 \\
\hline Very often & 39 & 68.42 \\
\hline \multicolumn{3}{|l|}{ Smartphone use } \\
\hline Never & 6 & 10.53 \\
\hline Rarely & 1 & 1.75 \\
\hline Sometimes & 6 & 10.53 \\
\hline Often & 5 & 8.77 \\
\hline Very often & 39 & 68.42 \\
\hline \multicolumn{3}{|l|}{ Downloaded app } \\
\hline No & 27 & 47.37 \\
\hline Yes & 30 & 52.63 \\
\hline \multicolumn{3}{|l|}{ Education } \\
\hline 9th-12th grade & 5 & 8.77 \\
\hline 2-y college & 8 & 14.04 \\
\hline 4-y college & 20 & 35.09 \\
\hline Graduate degree & 24 & 42.11 \\
\hline
\end{tabular}

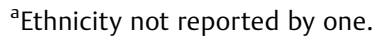

with version 2 ( $n=11), 10$ (91\%; 95\% exact CI, 59-100\%) used the app. Two of the three with both versions used the app. The tracking of symptoms (Health Tracker) and subsequent messaging from clinicians (My Inbox) were most accessed.

A total of 48 participants completed all of the 6 core acceptability items (easy, understandable, enjoy, helpful, amount of time, and satisfaction) to compute a total acceptability score of which the overall mean acceptability score was 
Table 2 Feature utilization: overall and by version

\begin{tabular}{|c|c|c|c|c|c|c|c|}
\hline \multirow[t]{3}{*}{ Feature } & \multirow{2}{*}{\multicolumn{2}{|c|}{$\begin{array}{l}\text { Overall } \\
(N=57)\end{array}$}} & \multicolumn{5}{|c|}{ Version } \\
\hline & & & \multicolumn{2}{|c|}{$\begin{array}{l}\text { Version } 1 \\
(N=43)\end{array}$} & \multicolumn{2}{|c|}{$\begin{array}{l}\text { Version } 2 \\
(N=11)\end{array}$} & \multirow{2}{*}{$\begin{array}{l}\begin{array}{l}\text { Both } \\
(N=3)\end{array} \\
N\end{array}$} \\
\hline & $N$ & $\%$ & $N$ & $\%$ & $N$ & $\%$ & \\
\hline Any use & 53 & 93 & 41 & 95 & 10 & 91 & 2 \\
\hline Calendar & 7 & 12 & 7 & 16 & 0 & 0 & 0 \\
\hline Community & 25 & 44 & 20 & 47 & 4 & 36 & 1 \\
\hline Dashboard & 24 & 42 & 19 & 44 & 3 & 27 & 2 \\
\hline Health Tracker & 49 & 86 & 39 & 91 & 8 & 73 & 2 \\
\hline Medical Diary & 18 & 32 & 15 & 35 & 3 & 27 & 0 \\
\hline My Inbox & 36 & 63 & 31 & 72 & 5 & 45 & 0 \\
\hline My Medications & 34 & 60 & 27 & 63 & 6 & 55 & 1 \\
\hline My Profile & 30 & 53 & 25 & 58 & 4 & 36 & 1 \\
\hline Nutrition & 26 & 46 & 21 & 49 & 5 & 45 & 0 \\
\hline Scrapbook & 23 & 40 & 19 & 44 & 4 & 36 & 0 \\
\hline Settings & 8 & 14 & 6 & 14 & 2 & 18 & 0 \\
\hline
\end{tabular}

Table 3 The proportion of participants indicating $>4$ on each acceptability item

\begin{tabular}{|c|c|c|c|c|c|c|c|c|c|}
\hline \multirow{3}{*}{$\begin{array}{l}\text { High } \\
\text { acceptability }\end{array}$} & \multirow{2}{*}{\multicolumn{3}{|c|}{ Overall $^{\mathrm{a}}$}} & \multicolumn{6}{|l|}{ Version } \\
\hline & & & & \multicolumn{3}{|c|}{ Version 1} & \multicolumn{3}{|l|}{ Version 2} \\
\hline & $\begin{array}{l}N \\
\text { respond }\end{array}$ & $\begin{array}{l}N \\
\geq 4\end{array}$ & $\%$ & $\begin{array}{l}N \\
\text { respond }\end{array}$ & $\begin{array}{l}N \\
\geq 4\end{array}$ & $\%$ & $\begin{array}{l}N \\
\text { respond }\end{array}$ & $\begin{array}{l}N \\
\geq 4\end{array}$ & $\%$ \\
\hline Easy & 51 & 45 & 88 & 40 & 35 & 87 & 9 & 8 & 89 \\
\hline Understandable & 51 & 48 & 94 & 40 & 38 & 95 & 9 & 8 & 89 \\
\hline Enjoy & 50 & 29 & 58 & 39 & 21 & 54 & 9 & 7 & 78 \\
\hline Helpful & 50 & 23 & 46 & 39 & 16 & 41 & 9 & 6 & 67 \\
\hline Amount of time & 51 & 45 & 88 & 40 & 34 & 85 & 9 & 9 & 100 \\
\hline Satisfaction & 51 & 34 & 67 & 40 & 25 & 62 & 9 & 7 & 78 \\
\hline Total acceptability & 48 & 31 & 65 & 37 & 23 & 62 & 9 & 6 & 67 \\
\hline
\end{tabular}

ancludes all reporting patients (version 1, version 2, both versions).

$24.8(\mathrm{SD}=4.2)$. The mean acceptability for version $1(n=37)$ was $24.5(\mathrm{SD}=4.5)$ and $25.5(\mathrm{SD}=3.7)$ for version $2(n=9)$. The proportion of participants indicating 4 or higher on each individual item of the acceptability scale is summarized in -Table 3. - Table 4 details the additional features scored

Table 4 The proportion of participants indicating $\geq 4$ on additional features for those items answered by at least 20 participants

\begin{tabular}{|l|l|l|l|}
\hline \multirow{2}{*}{ Item } & \multirow{2}{*}{$\begin{array}{l}\text { Number } \\
\text { answering }\end{array}$} & \multicolumn{2}{|l|}{ Score $\geq \mathbf{4}$} \\
\cline { 3 - 4 } & & $\mathbf{N}$ & $\%$ \\
\hline Messaging & 24 & 21 & 88 \\
\hline Doctor communication & 25 & 23 & 92 \\
\hline Graphs & 27 & 20 & 74 \\
\hline Resources & 21 & 15 & 71 \\
\hline Nutrition & 22 & 13 & 59 \\
\hline
\end{tabular}

if at least 20 participants responded to the item. Communicating with the doctor and graphing of symptoms were reported as the most acceptable features.

\section{Open-Ended Items}

Of 57 participants, 41 provided a response regarding their favorite feature of the app. The patient-clinician communication function was favored by 10 participants, followed by the symptom tracking function $(n=9)$. Five participants identified the daily medication reminder as a favorite. Suggestions from 24 participants regarding how to improve the app included: a more personalized application in which the user only tracks symptoms that were actually experienced $(n=5)$. The remaining suggestions from one or two participants each focused on further tailoring and trending features, better responsive design for the smartphone user, downloading and uploading functions, and integration with personal email. For the few participants who did not use the 
app remotely, reasons given were personal characteristics, not enough technology expertise $(n=4)$, and feeling too sick to use $(n=3)$.

\section{Clinician Results}

Three clinicians participated, all of whom were women and at least 30 years. One was a Doctor of Medicine and the other two, advanced registered nurse practitioner and registered nurse. Each had been in oncology practice for at least 6 years. All clinicians accessed the following features at least once: community, dashboard, inbox, medication and health alerts, my inbox, my profile, and participant dashboard. Two of the three clinicians accessed: participant record, settings, and symptom management. One of the clinicians used: medical diary and participant record. All three clinicians reported that the patient participants' use of the app enhanced the subsequent in-person visits.

\section{Open-Ended Clinician Responses}

Each clinician wrote a response with suggestions for improvement. Common to each suggestion was the difficulties caused by the version change during the study. Integration with the institutional electronic medical record was identified as essential for future use. Being able to visualize the self-care recommendation algorithm when alerted to a moderate or severe symptom was suggested.

\section{Discussion}

In a sample of patients actively undergoing treatment for GI cancer, and who had Internet access on a personal device, we found a high percentage of remote users and adequate acceptability with the iCancerHealth app. Our criteria for patient participant success in this pilot study were met.

A previous study ${ }^{28}$ of a Web-based intervention for cancer symptom and quality of life self-care in a more heterogeneous sample and without weekly prompting resulted in a much lower utilization rate (34\% vs. $93 \%$ this study). Our weekly reminders appear to have had an impact on the high access rates. Ruland et $\mathrm{al}^{27}$ in Norway reported a $77 \%$ unprompted access rate in a study of a Web-based program to support patients with prostate or breast cancer with symptom monitoring and self-care. That sample, recruited through advertisements in print media and one Web site, certainly was self-selected in contrast to our consecutive patient recruitment in the clinic. Similarly, in a recent pilot $^{33}$ of an app to monitor meals and self-care actions in participants with diabetes, 13 of 14 participants logged meal events over 30 days; it is unclear whether the participants were prompted in any way.

The tracking of symptoms (Health Tracker) and subsequent messaging from clinicians (My Inbox) were among the most highly utilized features. The value of the combined symptom reporting and communication with clinicians plus management information was evident in the acceptability scores plus the responses to open-ended queries regarding favorite feature. No other previous published study has detailed the acceptability of specific program features. How- ever, Ruland et $\mathrm{al}^{34}$ described feature usage details in which the discussion forum and messaging of nurses were most used. Messaging of clinicians was mainly automated in the iCancerHealth app because of the threshold function in which an alert to the clinician was sent for any symptom of a moderate to severe level. The community forum in our app was not used frequently and may be explained by the fact that the community included users outside of the clinical site and was not a secured component of the app.

Acceptability scores were higher than our preset threshold indicating adequate overall acceptability for an electronic program. The lowest scored core item was how helpful was it to use the app/Web site; however, only about half the participants answered this item at the last data collection time point.

All three clinicians reported high acceptability regarding how the app facilitated in-person interactions (telephone or in clinic) that occurred after patients used the app. Only one clinician gave high scores to the items related to ease of use, identifying appropriate issues, and symptom notifications promoting communication. Open-ended item reports from the clinicians indicated that "glitches" in the provider interface precluded higher ratings of the app. The request that the results of patient reports appear in the enterprise medical record is consistent with clinician feedback on recent app development for asthma symptoms. ${ }^{35}$ The sponsor's decision to change the app version in the middle of the trial was unfortunate and clearly not a best practice for future testing protocols.

Our findings are limited primarily by the inclusion criterion requiring personal access to an Internet-enabled device and the racial and ethnic homogeneity of the recruited sample. By requiring a personal device, we likely excluded those who were less technology savvy. Participants may have enrolled to receive compensation for time and effort. Our findings cannot be generalized beyond this typical, urban comprehensive cancer center population.

Future researchers are encouraged to include measures of specific features used and favored by participants. The impact of systematic reminders was substantial and should be considered when mounting new changes in patientreported outcomes. The high access rates, subsequent to telephone prompting, suggest the utilization of a Web-based program or app can be assured with such contact.

Our access rates and feature use findings are relevant to clinicians who promote Web-based monitoring of cancer symptoms, whether through an institutional vendor portal or a stand-alone system. Telephone follow-up by staff will likely enhance access rates.

\section{Conclusion}

The findings of this pilot study suggest that prompted patients with cancer who own Internet-enabled devices are willing and able to report symptoms and communicate with clinicians regularly in between clinic visits. These most frequently used features by patient participants also were reported as most acceptable among all features. Clinicians found the communication feature acceptable as well. 


\section{Clinical Relevance Statement}

Patient-clinician communication about symptoms in between clinic visits is highly utilized and valued by patients. A systematic approach to such a mechanism may result in more engaged patients and better symptom management.

\section{Multiple Choice Questions}

1. Web-based programs and apps for remote cancer symptom management have been tested and found to:

a. Reduce symptom distress.

b. Integrate well with enterprise medical record systems.

c. Interfere with patient-clinician communication.

d. Increase depressive symptoms.

Correct Answer: The correct answer is option a (As cited in the article introduction, the only correct outcomes in this list is option a).

2. Patient participants with gastrointestinal cancer favored which of the following iCancerHealth features?

a. Community forum.

b. Nutrition.

c. Messaging with doctor.

d. Calendar.

Correct Answer: The correct answer is option c (results in -Table 4; highest percent of high acceptability scores).

\section{Protection of Human and Animal Subjects}

This study was approved as minimal risk by the Dana-Farber Cancer Institute Institutional Review Board in Boston, Massachusetts.

\section{Funding}

Medocity, Inc. supported the first 3 months of the study through a contract to the Dana-Farber Cancer Institute.

\section{Conflict of Interest}

None.

\section{References}

1 Berry DL, Hong F, Halpenny B, et al. The electronic self report assessment and intervention for cancer: promoting patient verbal reporting of symptom and quality of life issues in a randomized controlled trial. BMC Cancer 2014;14(01):513

2 Berry DL, Hong F, Halpenny B, et al. Electronic self-report assessment for cancer and self-care support: results of a multicenter randomized trial. J Clin Oncol 2014;32(03):199-205

3 Reilly CM, Bruner DW, Mitchell SA, et al. A literature synthesis of symptom prevalence and severity in persons receiving active cancer treatment. Support Care Cancer 2013;21(06):1525-1550

4 Fann JR, Hong F, Halpenny B, Blonquist TM, Berry DL. Psychosocial outcomes of an electronic self-report assessment and self-care intervention for patients with cancer: a randomized controlled trial. Psychooncology 2017;26(11):1866-1871

5 Halfdanarson TR, Jatoi A. Oral cancer chemotherapy: the critical interplay between patient education and patient safety. Curr Oncol Rep 2010;12(04):247-252

6 Ruddy KJ, Partridge AH. Adherence with adjuvant hormonal therapy for breast cancer. Ann Oncol 2009;20(03):401-402
7 Hartigan K. Patient education: the cornerstone of successful oral chemotherapy treatment. Clin J Oncol Nurs 2003;7(6, Suppl):21-24

8 Spoelstra SL, Given BA, Given CW, et al. Issues related to overadherence to oral chemotherapy or targeted agents. Clin J Oncol Nurs 2013;17(06):604-609

9 Boucher J, Lucca J, Hooper K, Pedulla L, Berry D. A Structured nursing intervention to address oral chemotherapy adherence in patients with non-small cell lung cancer. Onc Nurs Forum 2015; 42(04):383-389

10 Cleeland CS, Zhao F, Chang VT, et al. The symptom burden of cancer: evidence for a core set of cancer-related and treatmentrelated symptoms from the Eastern Cooperative Oncology Group Symptom Outcomes and Practice Patterns study. Cancer 2013; 119(24):4333-4340

11 Hamilton J, Kruse H, Holcomb L, Freche R. Distress and psychosocial needs: demographic predictors of clinical distress after a diagnosis of cancer. Clin J Oncol Nurs 2018;22(04):390-397

12 Palma DA, Senan S, Tsujino K, et al. Predicting radiation pneumonitis after chemoradiation therapy for lung cancer: an international individual patient data meta-analysis. Int J Radiat Oncol Biol Phys 2013;85(02):444-450

13 Carlson LE, Waller A, Groff SL, Bultz BD. Screening for distress, the sixth vital sign, in lung cancer patients: effects on pain, fatigue, and common problems-secondary outcomes of a randomized controlled trial. Psychooncology 2013;22(08):1880-1888

14 American College of Surgeons. Cancer Program Standards 2012: Ensuring Patient-Centered Care. Chicago, IL: American College of Surgeons; 2012. Available at: http://www.facs.org/cancer/coc/ programstandards2012.pdf. Accessed October 20, 2018

15 Mooney K, Berry DL, Whisenant M, Sjoberg D. Improving cancer care through the patient experience: how to use patient-reported outcomes in clinical practice. American Society of Clinical Oncology educational book. American Society of Clinical Oncology Meeting; 2017;37:695-704

16 Vandyk AD, Harrison MB, Macartney G, Ross-White A, Stacey D. Emergency department visits for symptoms experienced by oncology patients: a systematic review. Support Care Cancer 2012;20(08):1589-1599

17 Heyn L, Ruland CM, Finset A. Effects of an interactive tailored patient assessment tool on eliciting and responding to cancer patients' cues and concerns in clinical consultations with physicians and nurses. Patient Educ Couns 2012;86(02):158-165

18 Boyes A, Newell S, Girgis A, McElduff P, Sanson-Fisher R. Does routine assessment and real-time feedback improve cancer patients' psychosocial well-being? Eur J Cancer Care (Engl) 2006;15(02):163-171

19 Velikova G, Booth L, Smith AB, et al. Measuring quality of life in routine oncology practice improves communication and patient well-being: a randomized controlled trial. J Clin Oncol 2004;22 (04):714-724

20 Salsman JM, Grunberg SM, Beaumont JL, et al. Communicating about chemotherapy-induced nausea and vomiting: a comparison of patient and provider perspectives. J Natl Compr Canc Netw 2012;10(02):149-157

21 Haynes RB, Ackloo E, Sahota N, McDonald HP, Yao X. Interventions for enhancing medication adherence. Cochrane Database Syst Rev 2008;(02):CD000011

22 Williams AF, Manias E, Walker R. Adherence to multiple, prescribed medications in diabetic kidney disease: a qualitative study of consumers' and health professionals' perspectives. Int J Nurs Stud 2008;45(12):1742-1756

23 Sommers RM, Miller K, Berry DL. Feasibility pilot on medication adherence and knowledge in ambulatory patients with gastrointestinal cancer. Oncol Nurs Forum 2012;39(04):E373-E379

24 Berry DL, Cunningham T, Eisenberg S, Wickline M, Hammer M, Berg C. Improving patient knowledge of discharge medications in an oncology setting. Clin J Oncol Nurs 2014;18(01):35-37 
25 Nielsen J.Usability 101: Introduction to Usability; 2012. Available at https://www.nngroup.com/articles/usability-101-introduction-tousability/. Accessed October 20, 2018

26 Wolpin S, Stewart M. A deliberate and rigorous approach to development of patient-centered technologies. Semin Oncol Nurs 2011;27(03):183-191

27 Ruland CM, Andersen T, Jeneson A, et al. Effects of an internet support system to assist cancer patients in reducing symptom distress: a randomized controlled trial. Cancer Nurs 2013;36(01):6-17

28 Berry DL, Blonquist TM, Patel RA, Halpenny B, McReynolds J. Exposure to a patient-centered, Web-based intervention for managing cancer symptom and quality of life issues: impact on symptom distress. J Med Internet Res 2015;17(06):e136

29 Mitchell PH, Ferketich S, Jennings BM; American Academy of Nursing Expert Panel on Quality Health Care. Quality health outcomes model. Image J Nurs Sch 1998;30(01):43-46

30 Tariman JD, Berry DL, Halpenny B, Wolpin S, Schepp K. Validation and testing of the Acceptability E-scale for web-based patientreported outcomes in cancer care. Appl Nurs Res 2011;24(01):53-58
31 Berry DL, Blumenstein BA, Halpenny B, et al. Enhancing patientprovider communication with the electronic self-report assessment for cancer: a randomized trial. J Clin Oncol 2011;29(08): 1029-1035

32 Berry DL, Halpenny B, Hong F, et al. The Personal Patient ProfileProstate decision support for men with localized prostate cancer: a multi-center randomized trial. Urol Oncol 2013;31(07): 1012-1021

33 Groat D, Soni H, Grando MA, Thompson B, Kaufman D, Cook CB. Design and testing of a smartphone application for real-time selftracking diabetes self-management behaviors. Appl Clin Inform 2018;9(02):440-449

34 Ruland CM, Maffei RM, Børøsund E, Krahn A, Andersen T, Grimsbø $\mathrm{GH}$. Evaluation of different features of an eHealth application for personalized illness management support: cancer patients' use and appraisal of usefulness. Int J Med Inform 2013;82(07):593-603

35 Rudin RS, Fanta CH, Predmore Z, et al. Core components for a clinically integrated mHealth app for asthma symptom monitoring. Appl Clin Inform 2017;8(04):1031-1043 